\title{
Dosagem de mistura asfáltica com CAP 50/70 modificado com borra de petróleo
}

Dosing asphalt mixture with CAP 50/70 modified with oil sludge

\author{
D. C. S. da Graça ${ }^{1 *}$, G. Cardoso ${ }^{1}$ e E. H. Cavalcante ${ }^{2}$ \\ ${ }^{1}$ Programa de Pós-Graduação em Engenharia Química/Laboratório de Desenvolvimento e Caracterização de \\ Materiais - LDCMat/Universidade Federal de Sergipe, 49100-000, São Cristóvão - SE, Brasil \\ ${ }^{2}$ Programa de Pós-Graduação em Engenharia Civil/Laboratório de Geotecnia e Pavimentação - \\ GEOPAV/Universidade Federal de Sergipe, 49100-000, São Cristóvão - SE, Brasil \\ *dagracadcs@yahoo.com.br \\ (Recebido em 15 de março de 2016; aceito em 22 de abril de 2016)
}

\begin{abstract}
Modificadores de ligantes asfálticos, tais como polímeros, borrachas de pneus moídos, borra oleosa de petróleo, dentre outros, vêm sendo estudados para melhor adequação destes às condições de uso. Contrariamente aos puros, os ligantes modificados apresentam comportamento reológico nãoNewtoniano. Neste estudo, a modificação do ligante asfáltico CAP 50/70 foi realizada utilizando-se 10\% e 20\%, em massa, de masterbatch's de borracha de pneus inservíveis com borra de petróleo, na proporção 15/85 (CM1) e 85/15 (CM2), caracterizadas reologicamente e em seguida obtidas misturas asfálticas por dosagem Marshall seguindo a Norma DNER - ME 043/95, e avaliados quanto ao comportamento da resistência à tração por compressão diametral (RTCD). Os resultados mostraram que as misturas com o CM1, de comportamento reológico de plástico ideal, apresentaram RTCD superior ao CAP 50/70, enquanto que as misturas com o CM2, de comportamento reológico pseudoplástico, apresentaram RTCD inferior ao CAP 50/70, contudo, superior ao estabelecido pela norma DNER-ME 138/94.
\end{abstract}

Palavras-chave: Ligante modificado, borra oleosa de petróleo, dosagem de ligante.

Modifiers asphalt binders, such as polymers, rubber tire minced, oily sludge oil, among others, have been studied to better adapt to these conditions of use. In contrast to CAP pure, modified ligands have nonNewtonian rheological behavior. In this study, modification of the asphalt binder CAP 50/70 was carried out using $10 \%$ and $20 \%$, by weight, of masterbatch's from rubber scrap tires with oil sludge in the proportion 15/85 (CM1) and 85/15 (CM2) following standard DNER - $043 \mathrm{ME} / 95$, and evaluated about of tensile strength by diametrical compression (TSDC) behavior. The results showed that the mix with CM1 that behaves as ideal plastics, showed lager TSDC than CAP 50/70, in another hand, the mix with CM2 that behaves as pseudoplastic showed TSDC below than CAP 50/70, however, lager than standard DNER-ME 138/94.

Keywords: modifield binders, oil sludge, dosing of binders.

\section{INTRODUÇÃO}

Em virtude do crescimento dos pesos por eixo dos veículos comerciais, da densidade de tráfego e de elevadas temperaturas climáticas têm sido cada vez mais necessário o uso de técnicas que contribuam para o melhor desempenho dos pavimentos assim como estudo e desenvolvimento de ligantes asfálticos modificados que proporcionem à mistura asfáltica melhor resistência ao revestimento da camada de rolamento dos pavimentos rodoviários [1-3]. Ressalta-se que os asfaltos convencionais, salvo para essas condições anteriormente mencionadas, ainda atendem satisfatoriamente a maioria das aplicações rodoviárias. A dificuldade reside na manutenção das características do CAP uma vez que estes são dependentes da composição química do petróleo do qual foi originado.

Dentre as formas de modificação, o uso de modificadores advindos de resíduos industriais para aumentar a resposta viscoelástico de ligante asfáltico e reduzir a sua susceptibilidade térmica têm sido largamente estudados [4-6]. 
Os pavimentos rodoviários são estruturas de múltiplas camadas, tendo como revestimento mistura de ligante asfáltico com agregados minerais graúdos, miúdos, filler, que se destina a dar maior conforto de tráfego ao usuário e resistir às ações climáticas, aumentando a vida útil do pavimento rodoviário. Portanto, essa mistura deve ser tanto quanto possível impermeável e resistente aos esforços de contato pneu-pavimento que variam conforme a carga e a velocidade dos veículos.

A temperatura é uma das variáveis de extrema importância para o controle do processo de obtenção de misturas asfálticas assim como do seu desempenho em serviço. Elevadas temperaturas de serviço, comuns na região nordeste do Brasil, favorecem o aparecimento de deformações permanentes no revestimento asfáltico dos pavimentos.

A resistência de mistura asfáltica à deformação permanente depende, além da temperatura, de fatores como rigidez do ligante asfáltico, dosagem do ligante na mistura, interação entre o agregado mineral e o ligante asfáltico e a porosidade da mistura asfáltica $[7,8]$.

Estudos científicos têm mostrado que a utilização de asfaltos modificados por polímeros propicia uma melhoria na resistência à formação de trilhas de roda e ao trincamento por fadiga, aumento da resistência ao envelhecimento, diminuição da susceptibilidade térmica, melhor característica coesiva e adesiva, elevação do ponto de amolecimento, dentre outros benefícios $[9,10]$.

De modo geral, o uso de resíduos industriais como matéria prima de novos processos tecnológicos tem sido prática cada vez mais comum. Também na modificação de ligantes asfálticos resíduos como borrachas de pneus, polímeros e agroindustriais têm sido empregados como agentes modificantes [11, 12], por este motivo o conhecimento do comportamento reológico decorrente das suas ações modificadoras torna-se importante para avaliar sua influência no comportamento reológico do ligante não modificado e nas propriedades de uso desses materiais na obtenção e aplicação de misturas asfálticas para revestimento de pavimento rodoviário $[13,14]$.

O estudo do comportamento de parâmetros reológicos em matérias asfálticos é importante para se estabelecer o processamento de misturas asfálticas e o controle da qualidade dos revestimentos dos pavimentos rodoviários, além de proporcionar um melhor entendimento do comportamento estrutural deste quando submetido ao tráfego $[15,16]$.

Os fluidos Newtonianos são aqueles cujo gráfico da tensão de cisalhamento versos taxa de cisalhamento, apresenta comportamento linear, passando pela origem. Enquanto que os fluidos não-Newtonianos apresentam gráficos de tensão versus taxa de cisalhamento, de modo geral, não apresentam comportamento linear, e quando linear, não passam pela origem.

Os ligantes asfálticos modificados, contrariamente aos puros, apresentam comportamento reológico não-Newtoniano o que requer atenção especial quando da sua dosagem em mistura asfáltica para revestimento de pavimento rodoviário [17].

Segundo Bernucci et al. [18], a dosagem de uma mistura asfáltica tem consistido até hoje na escolha, através de procedimentos experimentais, de um teor dito "ótimo" de ligante (teor de projeto), a partir de uma faixa granulométrica predefinida. Ou seja, dosar mistura asfáltica é definir por meio de experimentos laboratoriais a quantidade mais adequada de ligante asfáltico que irá servir como meio de ligação entre os agregados minerais, que foram previamente selecionados e classificados.

Para a determinação do teor de projeto de ligante, corpos de prova de mistura asfáltica na forma cilíndrica, trapezoidal, ou retangular, compactados por impacto, amassamento, vibração ou rolagem são obtidos. O teor de ligante pode variar a depender do sistema e do método de dosagem escolhido.

Neste estudo, amostras de ligante asfáltico CAP 50/70 modificado com $10 \%$ e $20 \%$ em massa de blendas de borracha de pneus inservíveis com borra de petróleo, na proporção 15/85 (CM1) e 85/15 (CM2) respectivamente, foram avaliadas quanto ao seu comportamento reológico na temperatura de processamento, à dosagem em mistura asfáltica pelo método Marshall, de acordo coma Norma DNER - ME 043/95 - Método de Ensaio - Misturas betuminosas a quente $[18,19]$ e à resistência mecânica por compressão diametral de acordo com a norma DNER - ME 138/1994 [20]. 


\section{MATERIAIS E MÉTODOS}

\subsection{Materiais}

Os materiais utilizados neste trabalho foram o cimento asfáltico de petróleo CAP 50/70, cedido pela EMURB - Aracaju/SE; a borra de petróleo de fundo de tanque de armazenamento como agente modificador de CAP, cedido pela Petrobras/UN-SEAL e a borracha de pneus inservíveis oriunda de unidade de recauchutagem de pneus da cidade de Itabaiana/SE, na forma de partículas inferiores a $0,595 \mathrm{~mm}$. Os agregados minerais empregados na obtenção das misturas asfálticas: graúdo (brita), miúdos (pó de pedra e areia), ambos de jazidas do estado de Sergipe, caracterizados quanto à granulometria e massa especifica segundo a norma DNIT-ES 031/2006 [21]. Como filler foi utilizado o cimento Portland CP-II-Z-32-RS, da marca POTY, do grupo Votorantim.

\subsection{Métodos}

\section{Modificação do ligante CAP 50/70}

Pré-misturas de pneus inservíveis e borra oleosa de petróleo (masterbatch), nas proporções de 15/85\% em massa, denominada M1 e 85/15\% em massa denominada M2, foram obtidas sob agitação mecânica de $40 \pm 2$ rpm e usada como agente modificante de ligante.

A modificação do ligante asfáltico se deu com a adição dos masterbatch's M1 e M2 nas concentrações de $10 \%$ e $20 \%$ em massa ao ligante asfáltico CAP 50/70, denominadas CM1 - 10 e CM1 - 20, CM2 - 10 e CM2 - 20, na temperatura de $170^{\circ} \mathrm{C}$, sob a mesma agitação da obtenção das pré-misturas, durante 90 minutos.

\section{Caracterização reológica do CAP 50/70}

O viscosímetro rotacional Brookfield é bastante utilizado na medida das propriedades do ligante asfáltico na temperatura de processamento e de compactação de misturas asfálticas, e a sua medida de viscosidade baseia-se na formulação teórica descrita na Tabela 1, cujos parâmetros dependem das características geométricas das hastes, dos elementos de rotação spindles, da câmara de amostra, da velocidade de rotação e da percentagem de deformação da mola do viscosímetro.

Tabela 1: Formulação teórica inerente ao cálculo da viscosidade com o viscosímetro Brookfield DV-III Ultra [22]

\begin{tabular}{|c|c|c|}
\hline Taxa de Cisalhamento $\left[\mathrm{s}^{-1}\right]$ & Tensão de Cisalhamento $\left[\mathrm{dy} / \mathrm{cm}^{2}\right]$ & $\begin{array}{c}\text { Viscosidade Dinâmica } \\
\text { [Poise] }\end{array}$ \\
\hline$\dot{\gamma}=\left(\frac{2 x R_{s}^{2}}{R_{c}^{2}-R_{s}^{2}}\right) \times \omega$ & $\tau=\frac{M}{2 \pi x R^{2} x L}$ & $\eta=\frac{\tau}{\dot{\gamma}}$ \\
\hline$\omega=\frac{2 \pi}{60} \times r p m$ & $\begin{array}{l}\mathrm{M}=[(\% \text { de deformação da mola }) \mathrm{x} \\
\text { (constante da mola) }] / 100\end{array}$ & \\
\hline
\end{tabular}

$\mathrm{R}_{\mathrm{c}}$ - raio da câmara de amostra [mm], $\mathrm{R}_{\mathrm{s}}$ - raio do spindle [mm], L - comprimento efetivo do spindle [mm], M - constante do equipamento [dy.cm], $\omega=$ velocidade angular, \% de deformação a mola, constante da mola, depende do modelo do viscosímetro, para o viscosímetro Brookfield DV-III Ultra é 7178 dy.cm.

Os valores da taxa de cisalhamento, tensão de cisalhamento e viscosidade são determinados pelas equações (1), (2) e (3), respectivamente, resultantes da simplificação das equações da Tabela 1, onde as constantes SRC e SMC dependem do spindle e da câmara de amostra e $T K$ do modelo do viscosímetro $S R C$ representa a constante da taxa de cisalhamento, SMC a constante do spindle e $T K$ a constante da mola do viscosímetro, constantes na Tabela 2. 


$$
\begin{aligned}
& \dot{\gamma}=\boldsymbol{S R C} \mathbf{x} \omega \\
& \tau=T K \times S M C \times S R C \times c o n s t . d a m o l a \quad\left[\mathrm{dy} / \mathrm{cm}^{2}\right] \\
& \eta=\left(\frac{100}{\omega}\right) \times T K \times S M C \times \text { const. da mola }[\mathrm{cP}]
\end{aligned}
$$

Tabela 2: Valores das constantes para os spidles do viscosímetro Brookfield DV-III Ultra

\begin{tabular}{llll}
\hline Spindles & SRC & SMC & TK \\
\hline 21 & 0,93 & 5 & 1 \\
27 & 0,34 & 25 & \\
\hline
\end{tabular}

As Tabelas 3 e 4 apresentam as características geométricas dos spindles e das câmaras e amostras para os spindles e viscosímetros utilizados no estudo [22].

Tabela 3: Características geométricas dos spindles utilizados no estudo

\begin{tabular}{lccc}
\hline Spindles & Diâmetro $[\mathrm{mm}]$ & Comprimento lateral [mm] & Comprimento efetivo [mm] \\
\hline 21 & 16,76 & 31,24 & 35,15 \\
27 & 11,76 & 33,02 & 39,29 \\
\hline
\end{tabular}

Tabela 4: Característica geométrica da câmara de amostra utilizada no Thermosel

\begin{tabular}{cc}
\hline Diâmetro [mm] & Comprimento [mm] \\
\hline 19,05 & 77,77 \\
\hline
\end{tabular}

\section{Dosagem da mistura asfáltica}

Para a determinação da dosagem de ligante nas misturas asfálticas em estudo foi empregado o procedimento de dosagem Marshall, o mais usado no Brasil, no qual primeiro determina-se a faixa granulométrica dos agregados [23]. Em seguida se caracteriza os agregados quanto à massa específica, de acordo com as normas NBR 6458/84 [24] para o agregado graúdo, e DNER-ME 194/98 [25] para os agregados miúdos.

O método de dosagem Marshall foi empregado, na forma recomendada pela norma NBR 12891 - Dosagem de Misturas Asfálticas pelo Método Marshall [26].

A moldagem dos corpos de prova se deu na forma cilíndrica, seguindo a norma DNER-ME $043 / 95$. Antes da realização das misturas os agregados miúdos e graúdos foram aquecidos a $110^{\circ} \mathrm{C} \pm 5^{\circ} \mathrm{C}$ em estufa, por $12 \mathrm{~h}$. Após a moldagem foram calculados, na temperatura ambiente, os parâmetros de determinação do teor de projeto de ligante asfáltico: volume de vazios (Vv), relação betume/vazios (RBV), Volume dos corpos de prova $(\mathrm{V})$, massa específica aparente $\left(\mathrm{d}_{\mathrm{M}}\right)$, densidade máxima teórica (DMT), volume de vazios com betume (VCB), vazios do agregado mineral (VAM), a ser utilizado conforme Bernucci et al. [18].

\section{RESULTADOS E DISCUSSÃO}

As Figuras 1a e 1b mostram o comportamento reológico do ligante asfáltico puro e modificado na temperatura de usinagem. Observa-se na Figura 1a, tendência de comportamento Newtoniano para o CAP 50/70 assim como os modificados CM1-10 e CM1-20, contrariamente aos modificados CM2 que apresentam comportamento tendendo a um plástico ideal e $\mathrm{o}$ aumento na concentração de CM2 elevou a viscosidade do ligante modificado como pode ser visto na Figura 1b, provavelmente influenciado pela maior concentração de borracha neste modificado. 


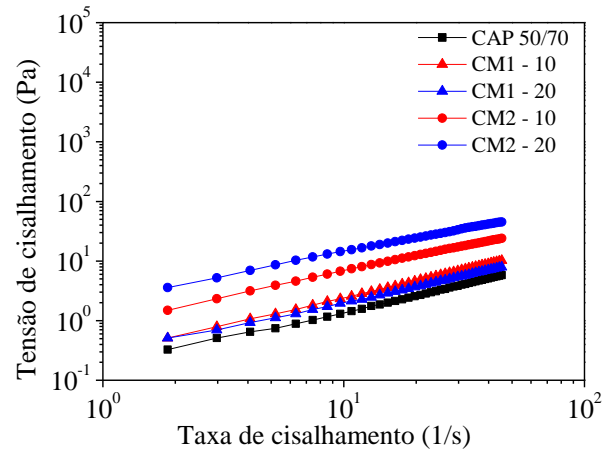

a)

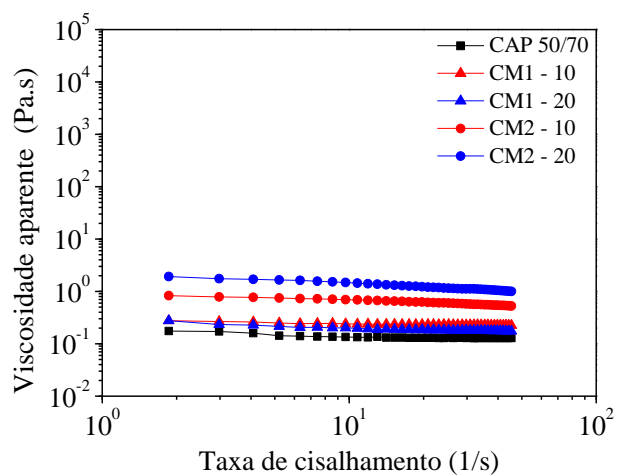

b)

Figura 1: Comportamento reológico em regime permanente do CAP 50/70 e ligantes modificados na temperatura de $170^{\circ} \mathrm{C}$ : (a) tensão cisalhamento versus taxa de cisalhamento e (b) viscosidade aparente versus taxa de cisalhamento

A Figura 2 mostra a distribuição granulométrica dos agregados minerais utilizados na obtenção das misturas asfálticas e a curva teórica da faixa C-DNIT obtida segundo as normas DNIT-ES 031/2006 e DNER-ME 035/95 [21, 24]. A definição da composição granulométrica na mistura asfáltica é feita por meio de ajustes quantitativos dos agregados que consiste na escolha da distribuição granulométrica para uma estrutura de agregados adequada ao uso desejado, garantindo maior empacotamento entre as partículas [27, 28, 29]. Para este estudo, optou-se por uma composição de mistura de agregado com $40 \%$ de brita, $30 \%$ de pó de pedra, $25 \%$ de areia e $5 \%$ de cimento Portland como material de enchimento, que comparativamente à curva da faixa C-DNIT [21], Figura 2, apresenta uma graduação granulométrica descontínua, na qual se buscou proporcionar com a grande concentração de finos um esqueleto mineral com poucos vazios e o agregado graúdo contribuindo para resistência à deformação permanente [30].

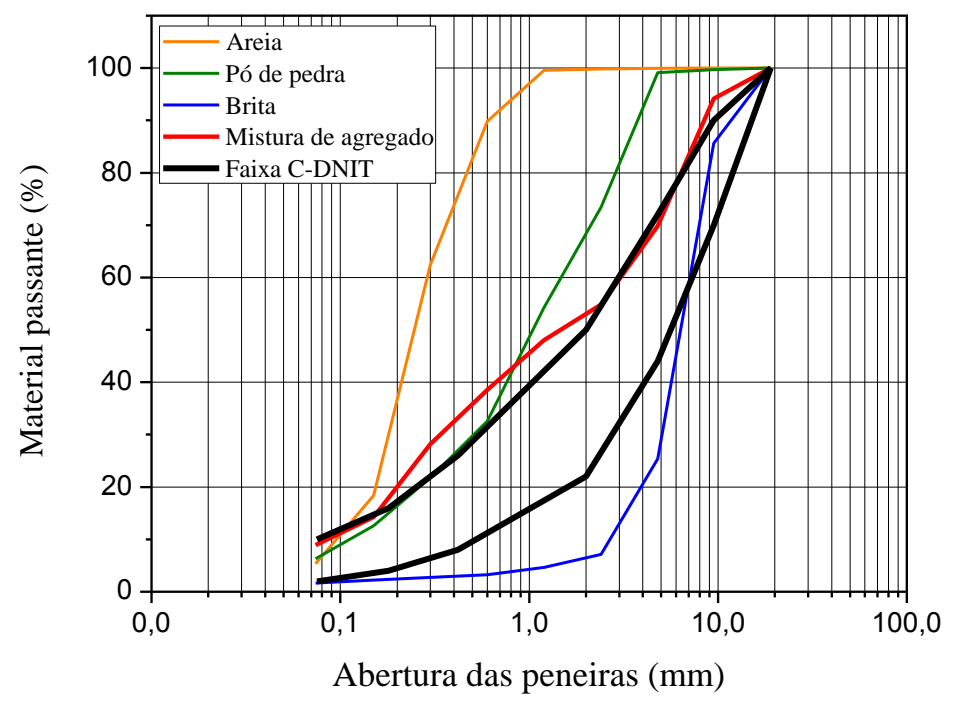

Figura 2: Curvas da distribuição granulométrica dos agregados utilizadas no estudo

As Figuras 3 e 4 mostram, respectivamente, para o ligante CAP 50/70 e modificado CM1-10, o comportamento do volume de vazios (Vv) e da relação betume/vazios (RBV) da mistura asfáltica, em função dos teores dos ligantes. Com base na norma DNIT-ES 031/2006 [21], para misturas asfálticas enquadradas na faixa C-DNIT, que estabelece valores de Vv e RBV versus ligante asfáltico, máximos $5 \%$ e $82 \%$ e mínimo $3 \%$ e $75 \%$, respectivamente, traçou-se as retas paralelas ao eixo das abscissas, com cujos pontos de intersecções com as retas Vv e RBV das misturas, determinou-se os teores máximo e mínimo de ligante, que a partir da sua média 
aritmética $\left[\left(\mathrm{x}_{2}+\mathrm{x}_{3}\right) / 2\right]$ obteve-se os teores de projeto de ligante para misturas asfálticas com $\mathrm{o}$ CAP 50/70 o valor de 5,5\% e para as misturas com o CAP modificado CM1-10, o valor de $5,3 \%$.

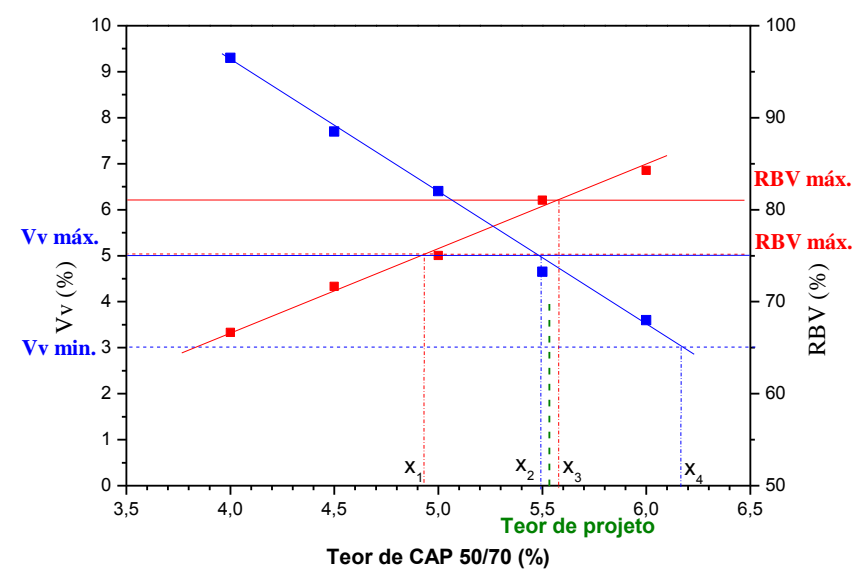

Figura 3: Curva de determinação do teor de projeto de ligante para o CAP 50/70 puro

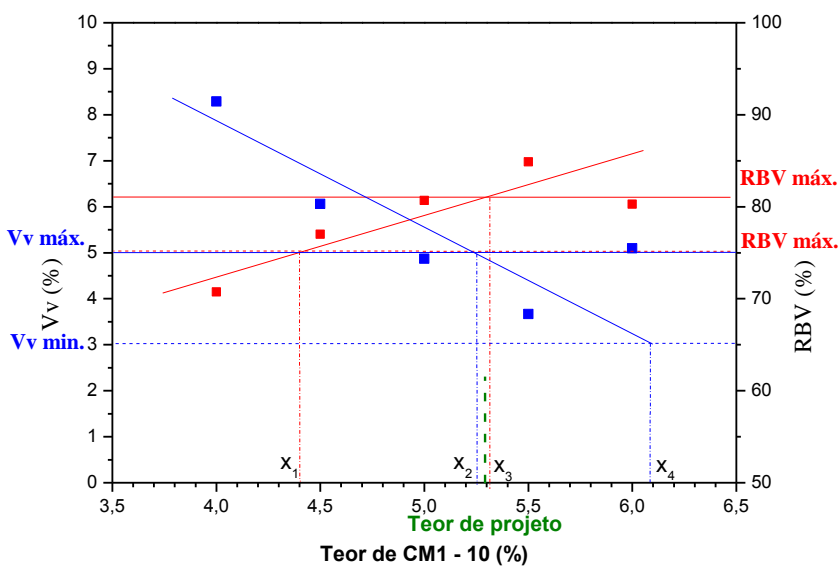

Figura 4: Curva de determinação do teor de projeto de ligante para a amostra CM1-10

A Figura 5 mostra o comportamento da resistência à tração por compressão diametral (RTCD) obtido a partir do valor médio de 03 (três) corpos de provas para cada mistura de ligante asfáltico em estudo, com os seus respectivos valores de desvios médios. Observa-se que o CAP modificado CM1 aumentou o valor da RTDC em relação ao CAP 50/70, enquanto que o CAP modificado CM2 diminuiu. O aumento do valor da RTCD do CAP modificado CM1 pode ser atribuído à menor viscosidade em comparação ao CAP modificado CM2. Contudo, observase que todas as misturas apresentaram valores de RTCD acima do valor estabelecido na norma DNIT-ES 031/2004 [21] para misturas asfálticas da faixa C-DNIT. 


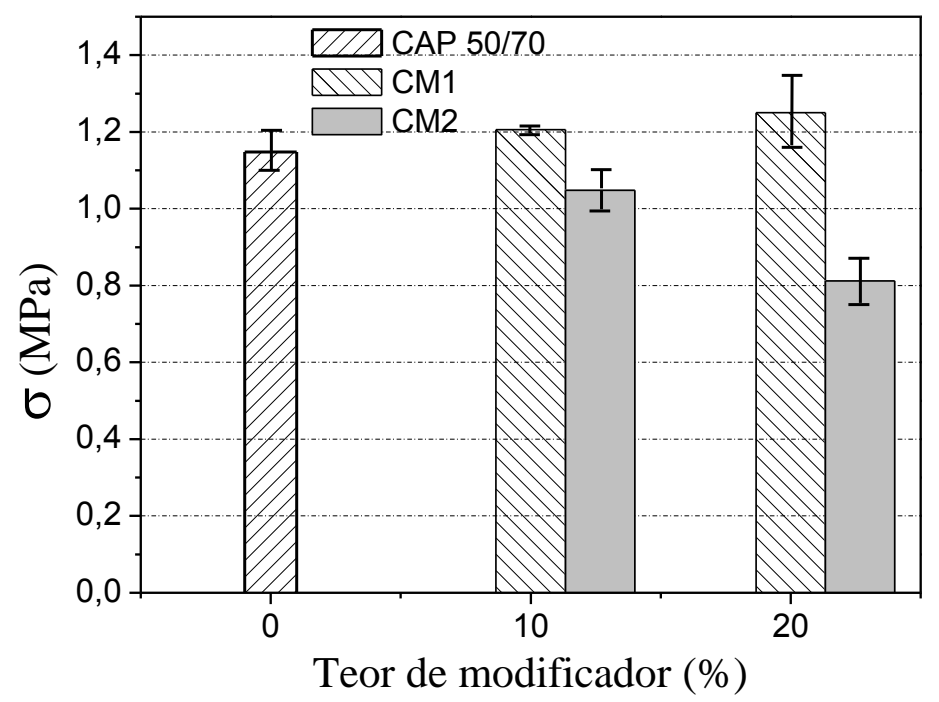

Figura 5: Comportamento da RTCD das misturas em função dos teores de agentes modificadores

\section{CONCLUSÃO}

É possível obter mistura asfáltica com o CAP 50/70 usando a borra de petróleo como modificador do ligante seguindo o procedimento de dosagem descrito na norma NBR 12891/1993 - Dosagem de misturas betuminosas pelo método Marshall [26], mesmo com os ligantes modificados apresentando comportamento reológico não-Newtoniano. $\mathrm{O}$ teor de projeto de ligante asfáltico obtido para o CAP $50 / 70$ de $5,5 \%$ e de $5,3 \%$ para o CAP modificado encontram-se na faixa de teores de projeto de ligante asfáltico estabelecidos para as misturas asfálticas [18]. Conclui-se que é possível utilizar dois resíduos de difícil degradação natural, borra oleosa de petróleo e borracha de pneus, na forma de masterbatch como agente modificador de ligante asfáltico e dosar suas misturas para revestimento de pavimento na forma convencional.

\section{AGRADECIMENTOS}

Os autores agradecem a CAPES pela concessão da bolsa de mestrado ao PEQ/UFS, à EMURB/Aracaju - SE e a Petrobras/UO-SEAL pelo fornecimento do ligante asfáltico e da borra oleosa de petróleo, respectivamente, utilizados no estudo, ao Laboratório de Geotecnia e Pavimentação (GEOPAV)/DEC-UFS pelo apoio na preparação e medidas das propriedades mecânicas das misturas asfálticas e a Petrobras UO-SEAL pela borra oleosa utilizada .

\section{REFERÊNCIAS BIBLIOGRÁFICAS}

1. Alatas T, Yılmaz M, Kök BV, Koral A. Comparison of permanent deformation and fatigue resistance of hot mix asphalts prepared with the same performance grade binders. Constr. Build Mater. 2012 Mai;30:66-72, doi: 10.1016/j.conbuildmat.2011.12.021.

2. Onofre FC, Castelo-Branco VTF, Soares JB; Faxina AL. Avaliação do efeito de ligantes asfálticos modificados na resistência à deformação permanente de misturas asfálticas densas. Transportes, doi: 10.4237/transportes.v21i3.685.

3. Yang X e You Z. High temperature performance evaluation of bio-oil modified asphalt binders using the DSR and MSCR tests. Constr. Build Mater. 2015 Fev;76:380-387, doi: 10.1016/j.conbuildmat.2014.11.063. 
4. Cardone F, Ferrotti G, Frigio F. Influence of polymer modification on asphalt binder dynamic and steady flow viscosities. Constr. Build. Mater. 2014 Out;71:435-443, doi: 10.1016/j.conbuildmat.2014.08.043.

5. Dias FJL, Santos PLG, Capitão SD. Mechanical performance of dry process fine crumb rubber asphalt mixtures placed on the Portuguese road network. Constr. Build. Mater. 2014 Set;73:247-254, doi: 10.1016/j.conbuildmat.2014.09.110.

6. Singh M, Kumar P, Maurya MR. Strength characteristics of SBS modified asphalt mixes with various aggregates. Constr. Build. Mater. 2013 Abr;41:815-823, doi: 10.1016/j.conbuildmat.2012.12.062.

7. Kaloush KE. Asphalt rubber: Performance tests and pavement design issues. Constr. Build. Mater. 2014; 67:258-264, doi: 10.1016/j.conbuildmat.2014.03.020.

8. Velmurugan A, Loganathan M, Gunasekaran EJ. Experimental investigations on combustion, performance and emission characteristics of thermal cracked cashew nut shell liquid (TC-CNSL) diesel blends in a diesel engine. Fuel. 2014 Ago;132:236-245, doi: 10.1016/j.fuel.2014.04.060.

9. Shirini B, Imaninasab R. Performance evaluation of rubberized and SBS modified porous asphalt mixtures. Constr. Build. Mater. 2016 Jan;107:165-171, doi: 10.1016/j.conbuildmat.2016.01.006.

10. Oda S, Fernandes JJL, Ildefonso JS. Analysis of use of natural fibers and asphalt rubber binder in discontinuous asphalt mixtures. Constr. Build Mater. 2012 Jan;26:13-20, doi: 10.1016/j.conbuildmat.2011.06.030.

11. Wei Y, Cheng F, Hou G, Sun S. Amphiphilic cellulose: Surface activity and aqueous self-assembly into nano-sized polymeric micelles. React. Funct. Polym. 2008 Mai;68:981-989, doi: 10.1016/j.reactfunctpolym.2008.02.004.

12. Kasemsiri P, Neramittagapong A, Chindaprasirt P. Curing kinetic, thermal and adhesive properties of epoxy resin cured with cashew nut shell liquid. Thermochim. Acta. 2015 Jan;600:20-27, doi: 10.1016/j.tca.2014.11.031.

13. Xu O, Xiao F, Han S, Amirkhanian SN, Wang Z. High temperature rheological properties of crumb rubber modified asphalt binders with various modifiers. Constr. Build. Mater. 2016 Mai;112:49-58, doi: 10.1016/j.conbuildmat.2016.02.069.

14. Liu H, Chen Z, Wang W, Wang H; Hao P. Investigation of the rheological modification mechanism of crumb rubber modified asphalt (CRMA) containing TOR additive. Constr. Build. Mater. 2014 Ago; 67:225-233, doi: 10.1016/j.conbuildmat.2013.11.031.

15. Jamshidi A, Golchin B, Othman HM, Turner P. Selection of type of warm mix asphalt additive based on the rheological properties of asphalt binders. J. Cleaner Prod. 2015 Fev;100:89 e 106, doi: 10.1016/j.jclepro.2015.03.036.

16. Singh M, Kumar P, Maurya MR. Strength characteristics of SBS modified asphalt mixes with various aggregates. Constr. Build. Mater. 2013 Abr; 41:815-823, doi: 10.1016/j.conbuildmat.2012.12.062.

17. Soenen H, Redelius P. The effect of aromatic interactions on the elasticity of bituminous binders. Rheol. Acta. 2014 Set; 53:741-754, doi: 10.1007/s00397-014-0792-0.

18. Bernucci LLB, Motta LMG, Ceratti e Fonseca JB. Pavimentação Asfáltica: Formação Básica para Engenheiros. Rio de Janeiro: PETROBRAS: ABED A, 2006.

19. DNER-DEPARTAMENTO NACIONAL DE ESTRADAS E RODAGENS. ME 043/95: misturas betuminosas a quente. IPR, Rio de Janeiro, 1995.

20. DNER - ME 138/1994 - Misturas Betuminosas - Determinação da Resistência à Tração por Compressão Diametral - DNER - Departamento Nacional de Estradas de Rodagem, IPR, Rio de Janeiro.

21. DNIT-ES 031/2006 - DEPARTAMENTO NACIONAL DE INFRAESTRUTURA DE TRANSPORTES. Pavimentos flexíveis - Concreto asfáltico - IPR, Rio de Janeiro, 2004.

22. Manual Brookfield DV - III Ultra - Programmable Rheometer, Operations Instructions n ${ }^{\circ}$ M/98-211C0310, Engineering Laboratories, Inc. Middleboro, USA.

23. DNER-ME 035/1995 - Especificação de material - DNER - Departamento Nacional de Estradas de Rodagem. Norma rodoviária. IPR, Rio de Janeiro.

24. NBR - 6458 - OUT/1984 - Agregado graúdo - Determinação de massa específica, massa específica aparente e absorção de água.

25. DNER - ME 194/1998 - Massa específica real - DNER - Departamento Nacional de Estradas de Rodagem. Instituto de Pesquisas Rodoviárias, Rio de Janeiro.

26. NBR 12891/1993 - Dosagem de misturas betuminosas pelo método Marshall.

27. Lopes M, Gabet T, Bernucci LLB, Mouillet V. Durability of hot and warm asphalt mixtures containing high rates of reclaimed asphalt at laboratory scale. Mater. Struct. 2015 Jan;48:3937-3948, doi: 10.1617/s11527-014-0454-9. 
28. Silvestre R, Medel E, García A, Navas J. Using ceramic wastes from tile industry as a partial substitute of natural aggregates in hot mix asphalt binder courses. Constr. Build. Mater. 2013 Abr;45:115-122, doi: 10.1016/j.conbuildmat.2013.03.058.

29. Vegas I, Ibañez JA, Lisbona A, Cortazar AS, Frías M. Pre-normative research on the use of mixed recycled aggregates in unbound road sections. Constr. Build. Mater. 2011 Jan;25:2674-2682, doi: 10.1016/j.conbuildmat.2010.12.018.

30. Motter JS, Miranda LFR, Bernucci LLB. Performance of Hot Mix Asphalt Concrete Produced with Coarse Recycled Concrete Aggregate. J. Mater. Civ. Eng. 2015 Mar; 27(11):04015030, doi: 10.1061/(ASCE)MT.1943-5533.0001284. 